\title{
Construction Solutions of Ordinary and Partial Differential Equations using the Analytical and Numerical Methods
}

\author{
Turkia Dhawi Alqurashi \\ Master of Mathematics (Numerical Analysis), College of Science, Shaqra University, Saudi \\ Arabia \\ Email: t.alkorashi@su.ed.sa
}

\begin{abstract}
:
In this paper we studied the solution of partial differential equations using numerical methods. The paper includes study of the solving partial differential equations of the type of parabolic, elliptic and hyperbolic, and the method of the net was used for the numerical nods, which represents a case of finite differences. We have two types of solution which are the internal solution and boundary solution. The internal solution is based on the internal nodes of the net. The boundary solution depends on the boundary nodes of the net, in addition to finding the analytical solution of the equations to compare the results. We also discussed solving the problem of Laplace, Poisson, for the importance of these equations in the applied side; Mat lab was used to find the values of tables for the values of border differences. We have derived a new formula for the solution of partial differential equations containing three independent variables.
\end{abstract}

Keywords: Regular Differential Equations, Partial Equations, Regular Differential Equivalent Solutions. 
درسنا في هذا البحث حل المعادلات التفاضلية الجزئية بالطرق العددية. تضمنت الورقة دراسة حل المعادلات التفاضلية الجزئية لنوع القطع المكافئ، و القطع الناقص، و القطعي، واستخدمت طريقة الثبكة للإيماءات العددية، والتي تمثل حالة الفروق المحدودة. لدينا نوعان من الحلول وهما الحل الداخلي وحل الحدود. يعتمد الحل الداخلي على العقد الداخلية للشبكة. بعتمد حل

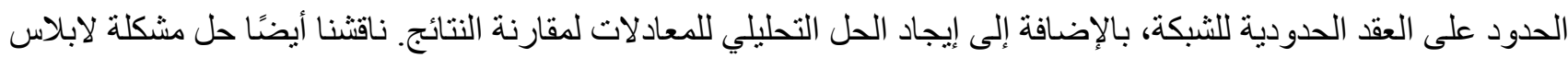

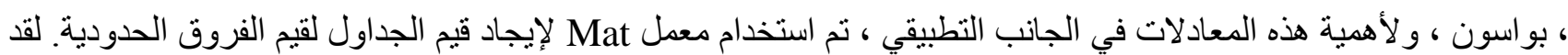
اشتقفنا صيغة جديدة لحل المعادلات التفاضلية الجزئية التي تحتوي على ثناثة متغير ات مستقلة.

الكلمات المفتاحية: المعادلات التفاضلية المنتظمة، المعادلات الجزئية، الحلول المكافئة التفاضلية المنتظمة.

\section{Introduction:}

The normal and partial differential equations are important in the rehabilitation and application of mathematics and physical, which are connected to sports and engineering science, the electrical and mechanical and sanctuary of the types of normal or partial differential equivalents (Essa, 2019).

There are no general sports methods to resolve differential equations, but there are some ways that can be circulated to a special group of differential equations (Othman \& Mahjob, 2016).The way of differences, which is more famous, and the way of the network is also used, where the essence of this method is to be the mainstream of the regions, where a separate set of points (the contract) is called the so-called network that replaces continuous diabetes to the network and is called the network ducts and as well as replace the derivatives in the differential equation Insurance of the differences in the differences of differences in the differential values of the differential equivalent system, network or the difference (the network equations or difference) and are called through the differences (Morton \& Mayers, 2005). The regular differential equation contains a single independent variable by the partial differential equation that has a number of independent variables such as temperature (which is based on two-way variables).

The study of the differential equations in the 18th century began to be reached by the researchers as an example, the Damlambeer, Lagrang, which appear in the issues related to the sound and heat, fluctuations of fluid and flexibility, 
the result of the importance of the role of the silver and the physical means of the squad and the awareness of a lot of mathematical and physical mattresses in a well-developed style. The findings of the numerical and analytical revenues of the normative and visual differences and the methods of building them. The number of ways that are based on the risk of irregular equation is easy to deal with it.

The current search seeks to the following:

- Solve and build regular differential equations using numerical roads.

- Solve the partial differential equations using analytical methods.

- Solve the normal differential equations using normal ways.

- Solve the partial differential equations using normal ways.

- Identify the uses of regular and analytical differential equivalents using numerical and analytical roads.

\section{Definition of differential equation:}

The equation is a differential equation if it contains derivatives for the approved variable for independent variables and the differential equation is the normal variable that is based on a single independent variable (Lippold, 1980).

\section{Differential equation:}

is a system of dollar that achieves differential equation and conveyor of derivatives, in the case of normal differential equations, can solve public and especially solutions, as general solutions to differential equations are solutions that contain many optional constants according to the differential level of the differential equation, the solutions obtained from selected values for optional reactions in the general solution, while individual solutions to differential equations are solutions that can not be obtained from the general solution (Hockney \& Eastwood, 2021).

\section{Regular differential equations:}

There are multiple varieties of regular differential equivalents:

A. The regular differential equation of the first level and you are following as the following format

$f(x, y, \grave{y})=0$ 
Where $\mathrm{F}$ is a function in the variables $(x, y, \grave{y})$ and the formula (1) is called as impressive formatting of normal differential equation, and the regular differential equation can be written from the first time (Essa, 2019):

$y^{`}=f(x, y)$

or $M(x, y) d x+N(x, y) d y=0$

The function $y=\phi(x)$ is available to the differential equation if the equation is achieved, which is when replacing the y and $y^{\prime}$ value in $\grave{\phi}(x), \phi(x)$ The equation is realized by the match, so equation $F(x, y, c)=0$ complete integration of differential equation and can be obtained through integration of differential curve integration curves, as the scheme of the differential equation process is called full-purpose (Morton \& Mayers, 2005).

The requirement is called $y=y_{0}, x=x_{0}$ or $y\left(x_{0}\right)=y_{0}=\varphi\left(x_{0}\right)$ with the primary condition of the differential equation (Mathews, 1992).

\section{Factive differential equations:}

The regular differential equation is called if the book is in the form of the following:

$$
f(x) d x+g(y) d y=0
$$

and the integration of ends of the equation we get the solution (Press et al., 1986).

- Numerical methods used to solve the normal differential equations of the first class These methods are on the equations that are formulated are

$\frac{d y}{d x}=f(x, y)$

the primary requirement $y\left(x_{0}\right)=y_{0}$, where numerical methods depend on the knowledge of the variable $y$ in the moment of starting $x_{0}$ and then launch this point step by step as we are estimated for $x_{0}+h$ and $y_{2}$ for $x_{1}+2 h$ where the growing h-Code is defined as $h=\frac{b-a}{M}$ (Othman \& Mahjob, 2016) 


\section{Oleer:}

This method of national reliability methods depends on giving a hard $h$ values small so that the Tyler series can be deleted $\frac{h^{2}}{2 !} y^{\prime \prime}(x)$ from the limit that contains the Tyler string of the Tyrler $(x+h)$ which Kalati is known as (Smith et al., 1985):

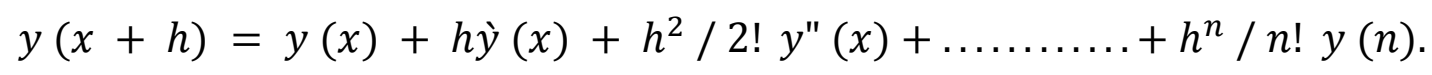

By deleting the border as of the $h^{2} / 2$ ! limit, $y(x+h)=y(x)+h y^{\prime}(x)=y(x)+h f(x, y)$ where we start from the point $\left(x_{0}, y_{0}\right)$ and compensation in the relationship above, the system

$y_{1}=y\left(x_{0}+h\right)=y\left(x_{0}\right)+h y^{\prime}\left(x_{0}\right)$

$y_{2}=y\left(x_{0}+2 h\right)=y\left(x_{0}\right)+2 h y^{`}\left(x_{0}\right)$

$y_{n+1}=y_{n}+h f\left(x_{n}, y_{n}\right) \quad n=0,1,2,3, \ldots \ldots M-1$

This is the general formula of law of the Auiler in the solution of differential equations

Example: The approximate solution is to the differential equation of $y^{\prime}=x+y$ where $y(0)=$ 0 , Take $h=0.2$ in the period $[0,1]$.

Solution

Using the Iuilel law and the requirement $y(0)=0$ where $x_{0}=0, y_{0}=0$ and the compensation of the law we find that

$$
\begin{aligned}
F\left(x_{n}, y_{n}\right)=x_{n}+y_{n} \quad, \quad x_{n}=x_{0}+n h=n h \\
y_{1}=y_{0}+0.2\left(x_{0}+y_{0}\right)=0 \\
y_{2}=y_{1}+0.2\left(x_{1}+y_{1}\right)=0+0.2(0.2+0)=0.4 \\
y_{3}=y_{2}+0.2\left(x_{2}+y_{2}\right)=0.4+0.2(0.4+0.04)=0.128 \\
y_{4}=y_{3}+0.2\left(x_{3}+y_{3}\right)=0.128+0.2(0.6+0.128)=0.274 \\
y_{5}=y_{4}+0.2\left(x_{4}+y_{4}\right)=0.48+0.2(0.8+0.489)=1.747 \\
y_{n+1}=y_{n}+0.2\left(x_{n}+y_{n}\right), x_{n}=0.2_{n}
\end{aligned}
$$

\section{The Rogen Metaway of the fourth rank:}

The previous OILE method in the solution of differential equations is used in practice as needs a small step (The value of the h must be small) for a reasonable accuracy, 
Tyler's triangle of the upper junior split as a general way to solve the differential equations because it needs many derivatives of the reservation $y(x)$ The Rogen Cuta method is preparing from the important ways to find the approximate solution to differential equations that help us get high accuracy with avoiding the need to derive the reservation $y(x)$ The unisex compensation is based $f(x, y)$ on selected points and therefore the general image of the equivalent of the rat mall of the 4th class is as follows (Mathews, 1992):

$$
y_{n+1}=\frac{y_{n+1}}{6\left(k_{1}+2 k_{n}+2 k_{n+1}+k_{4}\right)}
$$

Where:

$$
\begin{aligned}
& k_{1}=h f\left(x_{n}, y_{n}\right) \\
& k_{2}=h f\left(x_{n}+\frac{h}{2}, y_{n}+\frac{1}{2 k_{1}}\right) \\
& k_{3}=h f\left(x_{n}+\frac{h}{2}, y_{n}+\frac{1}{2 k_{2}}\right) \\
& k_{4}=h f\left(x_{n}+h, y_{n}+h k_{3}\right)
\end{aligned}
$$

Using the Rutan Kota 4 way finds the approximate solution to the issue of the primary condition :

$$
, y(0)=1, h=0.1 \quad 0 \leq x \leq 1 \quad, \quad y^{\prime}=-y+x+1
$$

Disentanglement

The equations of the Kota's cylinder can be written in the following picture:

$y_{n+1}=y_{n}+\frac{h}{6}\left(k_{1}+2 k_{2}+2 k_{3}+k_{4}\right)$

By applying the Rome Cacta equivalents where

$$
f(x, y)=-y+x+1
$$

$$
k 1=f\left(x_{n}, y_{n}\right)
$$

$$
\begin{aligned}
& k_{2}=f\left(x_{2}+\frac{h}{2}, y_{n}+\frac{h}{2 k_{1}}\right) \\
& k_{3}=f\left(x_{n}+\frac{h}{2}, y_{n}+\frac{h}{2 k_{2}}\right) \\
& k_{4}=f\left(x_{n}+h, y_{n}+h k_{3}\right)
\end{aligned}
$$


Which becomes as follows:

$$
\begin{aligned}
& k_{1}=-y+x+1 \\
& k_{2}=f\left(x_{n}+\frac{h}{2}, y_{n}+\frac{h}{2}\left(y_{n}+x_{n}+1\right)\right. \\
& k_{3}=f\left(x_{n}+\frac{h}{2}, y_{n}+\frac{h}{2}\left(\frac{h}{2}-1\right) y_{n}+\left(1-\frac{h}{2}\right) x_{n}+1\right) \\
& =\frac{h}{2}\left(1-\frac{h}{2}\right) y_{n}+\left(1-\frac{h}{2}\right) x_{n}+1 \\
& =\left(1-\frac{h}{2}\right)\left[\left(1-\frac{h}{2}\right)\left(x_{n}-y_{n}\right)+1\right]
\end{aligned}
$$

$K 4=\left(x_{n}+h, y_{n}+h k_{3}\right)$

$K 4=-y_{n}+h\left(1-\frac{h}{2}\left(1-\frac{h}{2}\right)\right)\left(x_{n}-y_{n}\right)+1+x_{n}+h+1$

For each $n=0,1,2,3,4, \ldots .9$

For example, when $n=0$ van:

$K 1=-y_{0}+x_{0}+1=0$

$K 2=\left(-0.95 y_{n}+0.95 x_{n}+1\right)=0.05$

$K 3=\left(-0.9525\left(x_{n}-y_{n}\right) y_{n}+1\right)=0.09525$

$K 4=-1-0.19525=0.0525$

And compensation of these values in the equation:

$y_{n+1}=y_{n}+\frac{h}{6}\left(k_{1}+2 k_{2}+2 k_{3}+k_{4}\right)$

We get on the: $y_{1}=y_{0}+\frac{h}{6}\left(k_{1}+2 k_{2}+2 k_{3}+k_{4}\right)$

$$
\begin{aligned}
& =0+\frac{0.1}{6}(0+2 \times 0.05+2 \times(1.09525)+(0.09525)) \\
& =1.0048375009
\end{aligned}
$$

Here we see the importance of the Roy Cot Ratio method as a nine way in solving the differential equations: 


\section{Classification of partial differential equations:}

The general state of the differential equations of the second level with two independent engines, the format (Press et al., 1986):

$F\left(x, y, U, U_{x}, U_{y}, U_{x x}, U_{x y}, U_{y y}\right)=0$

Partial derivatives. $U_{x}, U_{y}, U_{x x}, U_{x y}, U_{y y}$, an approved variable $\mathrm{u}$, independent variables $X, y$ so that any translation of the equation to the function, that the graph of the solution represents $U(X, Y)$ knows the solution of differential equation that the roof function for this function. The equation is said to be linear if the function and all their first class and derivatives are inherited. The linear equation can be written following:

$A(x, y) x x+B(x, y) u_{x y}+C(x, y) u_{y y}+D(x, y) u_{x}+E(x, y) u_{y}+F(x, y) u=R(x, y)$

The amount of $D=B 2(x, y)-4((x, y) C(x, y))$ is distributed to (2) the equation of the $\mathrm{D}$, so there are three categories of the sealing of the two-party partner difference (2) (Mathews, 1992):

1- $D>0$ If it is (Hyperbolic) a differential equation of the type of olive.

2- $D=0$ If it is (parabolic) a differential equation of equivalent type.

3- $D<0$ If Elliptic is a differential equation of the type of counterpart.

Definition: The issue of a special solution to the differential equation that certain primary conditions, called the Qushi issue. Differential equations are one of the most important math branches in their applications, and the differential equation of the difference, a particular function must be found, so that we can put a law whose description is given as a certain phenomenon or a process (Farlow, 1993).

Definition: Solution Question of Queshi Type: $(F, X, Y)$ is a solution to the equation given to the Y-Ox form, XP. Choose a solution from an infinite group requires the prelimination of preliminary conditions and borders. In this work B and the limits of R Robent, we are taking a continuous and closed area, therefore, a solution to the differential equation must be found if the primary and border conditions are given.(Othman \& Mahjob, 2016).

\section{Differential terms of gender:}

When studying the rapid operations of different physical nature (The vibrations, heat transfer, spread) usually comes from the equality of the core type. To take the equation of Labul: $\Delta u=0$ 
$\Delta u=\operatorname{div} \operatorname{grad} u=\frac{\partial^{2} u}{\partial x_{1}^{2}}+\frac{\partial^{2} u}{\partial x_{2}^{2}}+\cdots+\frac{\partial^{2} u}{\partial x_{n}^{2}}=0$

The Tastem function is called in the region if it is continuing in this area with its derivatives until the second rank and achieves the equation of Labuls (Essa, 2019).

\section{The method of differences specified to round derivatives:}

the idea of the specific difference method, or network method is: in the R / Diamond area (the 98bit) area is created, which is limited to BM borders to round the b limit (B) RE (Figure 2) (Press et al., 1986).

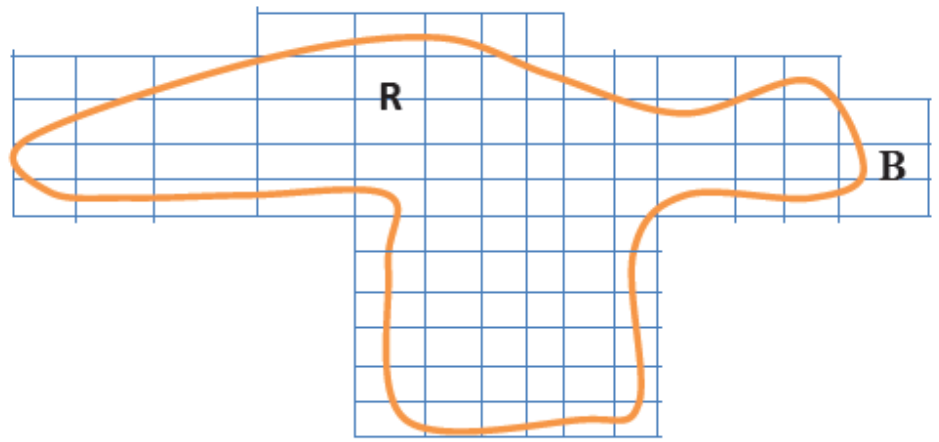

2. The differential equation is known to be the field of network replacing the equation of differences (to rubble with the specific derived.

3. The internal scale and the border of the GH areas and board bands are connected BH by the system of linear or non-linear equations system $(x, y) \rightarrow u\left(x_{i}, y_{j}\right)$. So the continuous solution is made through a selection of separate values from the network field. To take the network on the surface $(X, Y)$ and the following field of the network:

$x_{i}=x_{0}+i h$,

$y_{j}=y_{0}+j k$,

Where: $0 \leq i \leq N, 0 \leq j \leq M$

To round the first derived and second for the $X$ variable we use the Ticker microwave for Magitarin and Callati (Morton \& Mayers, 2005):

$u\left(x_{i \pm 1}, y_{j}\right)=u\left(x_{i} \pm h, y_{j}\right)=u\left(x_{i}, y_{j}\right) \pm h \frac{\partial u}{\partial x}\left(x_{i}, y_{j}\right)+\frac{h^{2}}{2} \frac{\partial u}{\partial x^{2}}\left(x_{i}, y_{j}\right) \pm$

$\pm \frac{h^{3}}{6} \frac{\partial^{3} u}{\partial x^{3}}\left(x_{i}, y_{j}\right)+\frac{h^{4}}{24} \frac{\partial^{4} u}{\partial x^{4}}\left(\bar{x}_{i}, y_{j}\right)$ 
$\mathrm{R}$ and region B form-2-network network border $\bar{x} \in x_{i}+\theta h, 0<\theta<1$ Take the first two and seconds of the series above and then find the solution for the first duration of the variable $X$

$$
\begin{gathered}
\frac{\partial u}{\partial x}\left(x_{i}, y_{j}\right)=\frac{u\left(x_{i+1}, y_{j}\right)-u\left(x_{i}, y_{j}\right)}{h}+O(h)= \\
\frac{u\left(x_{i}, y_{j}\right)-u\left(x_{i-1}, y_{j}\right)}{h}+O(h) \approx \frac{u\left(x_{i+1}, y_{j}\right)-u\left(x_{i-1}, y_{j}\right)}{2 h}+O\left(h^{2}\right)
\end{gathered}
$$

We use the same way to find the second derivative with note that it will appear first and derived up in the first derived from the first derived from the differences to get:

$$
\frac{\partial^{2} u}{\partial x^{2}}\left(x_{i}, y_{j}\right)=\frac{u\left(x_{i+1}, y_{j}\right)-2 u\left(x_{i}, y_{j}\right)+u\left(x_{i-1}, y_{j}\right)}{h^{2}}+O\left(h^{2}\right)
$$

$\frac{\partial u}{\partial y}, \frac{\partial^{2} u}{\partial y^{2}}$ variable $y$

In the same way, we can find the first and secondary

$\frac{\partial u}{\partial y}, \frac{\partial^{2} u}{\partial y^{2}}$ variable $y$

Derivatives that the basis of the selection of the network size depends on the volume of the error in the round of differential equation by the specified difference method. However, this approach is in a hard or investigative process. Therefore, to calculate the procedures, a double conversion map is used: The solution is built on a set of steps from $h, \mathrm{~h} / 2$ and $\mathrm{h} / 4$ solution of partial differential equations by the limited level of expenses lead to collective accounts of unified procedures and therefore easily combine these methods. Equipment for Labuls and Fiso in the specified Limited Remember that the equation of Poisson (4) $\Delta u=-f$

Its equation (Mathews, 1992):

$\frac{\partial^{2} \mathrm{u}}{\partial \mathrm{x}^{2}}+\frac{\partial^{2} \mathrm{u}}{\partial \mathrm{y}^{2}}=-f(x, y)$

In the practical side, many templates are used to build schemes for correspondence. To now write bulk bulk to the elements of the neon:

$\frac{u_{i+1, j}-2 u_{i, j}+u_{i-1, j}}{h^{2}}+\frac{u_{i, j+1}-2 u_{i, j}+u_{i, j-1}}{k^{2}}=-f(x, y)$

For the Lasel to the Florip, the formula will be similar, but $h=k$ and the following: 


$$
\begin{gathered}
\frac{u_{i+1, j}-2 u_{i, j}+u_{i-1, j}}{h^{2}}+\frac{u_{i, j+1}-2 u_{i, j}+u_{i, j-1}}{h^{2}}=0 \\
\frac{u_{i+1, j}-4 u_{i, j}+u_{i-1, j}+u_{i, j+1}+u_{i, j-1}}{h^{2}}=0 \\
u_{i, j}=\frac{1}{4}\left[u_{i+1, j}+u_{i-1, j}+u_{i, j+1}+u_{i, j-1}\right] \ldots \ldots \ldots \ldots \ldots \ldots \ldots \ldots \ldots
\end{gathered}
$$

It can be noted that the function $\left(x_{i}, y_{j}\right)$ is the solution to the Labulas in the node $(I, J)$, average the neighboring contract and the differences are selected in the following plans (Lippold, 1980):

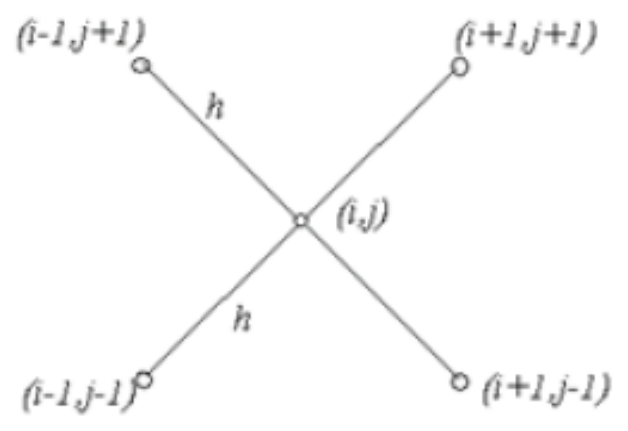

Form (4) scheduled selection charts.

For the Laselax to the Royal Region of the Group and the above scheme will be by the following equation (Smith et al., 1985):

$u_{i, j}=\frac{1}{4}\left[u_{i+1, j+1}+u_{i+1, j-1}+u_{i-1, j-1}+u_{i-1, j+1}\right]$

To take a problem to Drilcell's problem to the Lailpace:

$\Delta u=0, P(x, y) \in G, u(P)=\varphi(P), P \in B$

Continuous function $\varphi(P)$ so that in the case of the source network, the two decades are called one distance on the contractor, the contract of the inner part of the internal contract is called. The contract that has a least one internal negotiator is called the border contract of the first type. The equation solution (8) through the way of the differences limited, according to equation (6), 
We get the assembly system for each internal node of the contract distribution area $\left(x_{i}, y_{j}\right) \in S h$ of the border condition in (8) We find: $u(A)=\varphi(A)$ (9) Where the A venue of the border B.

Solve the equivalent partial equivalent equity using network method: To take the issue of heat: to find the function $u(x, t)$ that is equal: (Morton \& Mayers, 2005)

$$
\frac{\partial u}{\partial \mathrm{t}}=a^{2} \frac{\partial^{2} u}{\partial x^{2}}
$$

With $\quad$ conditions: $\quad U(x, 0)=f(x),(0<x<s)$

For border terms : $u(0, t)=\varphi(t), u(s, t)=\psi(t)(t>0)$ To find the approximate solution for the previous issue by the network method, take a rectangular network of the contract in the tape $0 \leq x \leq s, t \geq 0$ The intersection points are two groups of parallel lines: $x=i h,(i=$ $0,1, \ldots), t=j k,(j=0,1, \ldots)$

Now to put: $x i=i h, t_{i}=j k, u_{i j}=u\left(x_{i}, t_{j}\right)$,

Now to find approximate replacement at each internal node $\left(x_{i}, t_{j}\right)$ for second subscription $\frac{\partial^{2} u}{\partial x^{2}}$ $\frac{\partial^{2} u}{\partial x^{2}} \approx \frac{u_{i+1, j}-2 u_{i, j}+u_{i-1, j}}{h^{2}}$

Approximate replacement at each internal node $\left(x_{i}, t_{j}\right)$ for the first subscription:

$$
\frac{\partial \mathrm{u}}{\partial \mathrm{t}} \approx \frac{u_{i, j+1}-u_{i, j}}{k}
$$

To compensate for these arrests in heat equation, we find:

$$
\frac{u_{i, j+1}-u_{i, j}}{k}=a^{2} \frac{u_{i+1, j}-2 u_{i, j}+u_{i-1, j}}{h^{2}}
$$

The first difference chart is clear, the second is implicit. So we will get the valuable values that include values of the solution in four holding and accuracy of the accuracy of $(k+h)$ to put $\rho=$ $\frac{a^{2} k}{h^{2}}$, turn the final equation to the following form:

$u_{i, j}+1=1-2 \rho u_{i j}+\rho u_{i}+(1, j)+u_{i}-(1, j)$

At choice number (p) in equal above (Essa, 2019): 
- The precision in the replacement of the differential equation must be the most important of the differences.

- The stable difference must be stable

- that the previous formula is stable if it is $0<\rho \leq \frac{1}{2}$

As a simplified form of previous equation $\quad \rho=\frac{1}{2}$

$u_{i, j+1}=\frac{u_{i+1, j}+u_{i-1, j}}{2}$

When $\rho=\frac{1}{6}$

$$
u_{i, j+1}=\frac{1}{6} u_{i-1, j}+4 u_{i, j}+u_{i+1, j}
$$

Example:

Using the equation of the differences (10) Trust the round of the equation equivalent: $u t=8 u_{x x}$ $u(x, 0)=13 \sin \frac{\pi}{2} x \quad, 0 \leq x \leq 2$

And

$$
u(0, t)=u(0, t)=0,0 \leq t
$$

The percentage of the changer $X$ is selected $(h=0.25)$ and using the equation of the difference

(10) that is $(P=1 / 2)$ for the variable $(\mathrm{t})$, we find that $k=\frac{p h^{2}}{a^{2}}: U_{i, j+1}=$

$\frac{u_{i, j+1}+u_{i, j-1}}{2}$ this is the leads

$U_{i, 1}=\frac{u_{i+1,0}+u_{i-1,0}}{2}$

$U_{1,1}=\frac{u_{2,0}+u_{0,0}}{2}=\frac{1}{2}(9.1924+0)=4.5962$

$U_{2,1}=\frac{u_{3,0}+u_{1,0}}{2}=\frac{1}{2}(12.0104+4.9749)=8.4927$

So be table values function at some

$$
i=1,2,3,4,5,6,7,8 \& j=1,2,3,4,5,6,7,8
$$

Analytical analysis of the equal state equal to the comparison: 
Academic Journal of Research and Scientific Publishing | Vol 3 | Issue 33

Publication Date: 5-1-2022 ISSN: 2706-6495

$$
\tilde{u}(x, t)=\sum_{n=1}^{\infty} e^{-2 \pi^{2} n t} B_{n} \sin \frac{\pi n}{2} x
$$

As

$$
B_{n}=\frac{2}{2} \int_{0}^{2} 13 \sin \frac{\pi}{2} x \sin \frac{\pi n}{2} x d x \neq 0
$$

At $n=1$

$$
B_{1}=13 \int_{0}^{2} \sin ^{2} \frac{\pi}{2} x d x=\frac{13}{2} \int_{0}^{2}(1-\cos \pi x) d x=\frac{13}{2}\left[x-\frac{1}{\pi} \sin \pi x\right]=13
$$

And this is finding : $\tilde{u}(x, t)=13 e^{-2 \pi^{2} t} \sin \frac{\pi}{2} x \quad$ (Smith et al., 1985).

In the last two lines of the table below the valid solution values are given to the issue and precision

\begin{tabular}{|c|c|c|c|c|c|c|c|c|c|c|}
\hline & $i$ & 0 & 1 & 2 & 3 & 4 & 5 & 6 & 7 & 8 \\
\hline$j$ & $t_{j} x_{i}$ & 0,0 & 0,25 & 0,5 & 0,75 & 1,0 & 1,25 & 1,5 & 1,75 & 2,0 \\
\hline 0 & 0,0 & 0 & 4,9749 & 9,1924 & 12,0104 & 13,0 & 12,0104 & 9,1924 & 4,9749 & 0 \\
\hline 1 & 0,0039 & 0 & 4,5962 & 8,4927 & 11,0962 & 12,0104 & 11,0962 & 8,4927 & 4,5962 & 0 \\
\hline 2 & 0,0078 & 0 & 4,2464 & 7.8462 & 10.2516 & 11.0962 & 10.2516 & 7.8462 & 4,2464 & 0 \\
\hline 3 & 0,0117 & 0 & 3.9231 & 7,2490 & 9,4712 & 10.2516 & 9.4712 & 7,2490 & 3.9231 & 0 \\
\hline 4 & 0,0156 & 0 & 3,6245 & 6.6972 & 8.7503 & 9,4712 & 8.7503 & 6,6972 & 3.6245 & 0 \\
\hline 5 & 0,0195 & 0 & 3,3486 & 6,1874 & 8,0842 & 8,7503 & 8,0842 & 6,1874 & 3,3486 & 0 \\
\hline 6 & 0,0234 & 0 & 3,0937 & 5,7164 & 7,4689 & 8,0842 & 7,4689 & 5,7164 & 3.0937 & 0 \\
\hline 7 & 0,0273 & 0 & 2,8582 & 5,2813 & 6,9003 & 7,4689 & 6,9003 & 5,2813 & 2,8582 & 0 \\
\hline 8 & 0,0312 & 0 & 2,6407 & 4,8793 & 6,3751 & 6,9003 & 6,3751 & 4,8793 & 2,6407 & 0 \\
\hline \multicolumn{2}{|c|}{$\tilde{u}(x ; 0,0312)$} & 0 & 2,6873 & 4,9655 & 6,4877 & 7,0223 & 6,4877 & 4,9655 & 2,6873 & 0 \\
\hline \multicolumn{2}{|r|}{$|\bar{u}-u|$} & 0 & 0,0466 & 0,0862 & 0,1126 & 0,1220 & 0,1126 & 0,0862 & 0,0466 & 0 \\
\hline
\end{tabular}
factor $|\tilde{u}-u|$ at $\quad . t=0.0312$ Here can find the rest of the values by the following table (Lippold, 1980):

The method of advantages of Koshi:

This method is used to resolve non-linear differential equations on the following image (Farlow, 1993):

$f\left(x, y, z, \frac{\partial z}{\partial x}, \frac{\partial z}{\partial y}\right)=0$ 
Which can be written on the picture:

$$
f(x, y, z, p, q)=0: p=\frac{\partial z}{\partial x} \quad, q=\frac{\partial z}{\partial y}
$$

No one of the five-year-old category $\{y(t), x(t), p(t), q(t), z(q)\}$ Check the equation: $\mathrm{C}$ on the curve $(x, y, z)$ The sector is at point $z^{\prime}(t)=p(t) x^{\prime}(t)+q(t) y^{\prime}(t), \quad x=x(t), y=y(t), z=z(t) \quad$ for each values $\mathrm{T}$ on a convenient and contestant and if $(I)$ at the bottom of the curve $C$. The prime The sector is a distinctive sector and the diverse equations of the sector are as derived as follows:

Total inspec to $z$ (Hockney \& Eastwood, 2021):

$d z=\frac{\partial z}{\partial x} d x+\frac{\partial z}{\partial y} d y$

Or $\quad d z=p d x+q d y$

Where $p, q$ check the equation

By ending the equation (3) for $\mathrm{P}$ :

$d x+\frac{d q}{d p} d y=0 \quad \Rightarrow \quad \frac{d q}{d p}=-\frac{d x}{d y}$

By ending the equation (2) for $\mathrm{P}$ :

$f_{p}+\frac{\partial f}{\partial q} \frac{d q}{d p}=0 \quad \Rightarrow \frac{d q}{d p}=-\frac{f_{p}}{f_{q}}$

This is included:

$\frac{d x}{f_{p}}=\frac{d y}{f_{q}} \quad \Rightarrow \quad d x=\frac{f_{p}}{f_{q}} d y$

And compensation for $\mathrm{dx}$ in the equation (3):

$$
d z=p \frac{f_{p}}{f_{q}} d y+q d y \quad \Rightarrow \quad d z=\left(\frac{p f_{p}+p f_{q}}{f_{q}}\right) d y
$$

Any: $\frac{d z}{p f_{p}+q f_{q}}=\frac{d y}{f_{q}}$ 
Of the two equation (6), (7) we find that:

$\frac{d x}{f_{p}}=\frac{d y}{f_{q}}=\frac{d z}{p f_{p}+q f_{q}}$

So on the eligibility of the distinctive sector of the $\grave{x}(t), \grave{y}(t), \grave{z}(t) \quad$ should be proportional to $f_{p}, f_{a}, p f_{p}+q f_{a}$ respectively, and if we choose the medium $(t)$ on the following image (Mathews, 1992):

$\grave{x}(t)=f_{p} \quad \grave{y}(t)=f_{a}$

Mortal:

$$
\grave{z}(t)=p f_{p}+q f_{a}
$$

On the ability to distinguish the sector is a job in $\mathrm{T}$ and so on:

$\dot{p}(t)=\frac{\partial p}{\partial x} \frac{d x}{d t}+\frac{\partial p}{\partial y} \frac{d y}{d t}$

Of the two equations in (9) we find that:

$\dot{p}(t)=\frac{\partial p}{\partial x} f_{p}+\frac{\partial p}{\partial y} f_{q}$

But:

$\frac{\partial p}{\partial y}=\frac{\partial}{\partial y}\left(\frac{\partial z}{\partial x}\right)=\frac{\partial}{\partial x}\left(\frac{\partial z}{\partial y}\right)=\frac{\partial}{\partial x}(q)$

Wherefore:

$$
\dot{p}(t)=\frac{\partial p}{\partial x} f_{p}+\frac{\partial q}{\partial x} f_{q}
$$

By the end of the equation (2) for $X$ :

$f_{x}+p f_{z}+f_{p} \frac{\partial p}{\partial x}+f_{q} \frac{\partial q}{\partial x}=0$

Or $f_{p} \frac{\partial p}{\partial x}+f_{q} \frac{\partial q}{\partial x}=-\left(f_{x}+p f_{z}\right)$

Of the two equations in (11), (12) we find that:

$p^{\prime}(t)=-\left(f_{x}+p f_{z}\right)$ 
As we find that:

$\dot{q}(t)=\frac{\partial q}{\partial x} \frac{d x}{d t}+\frac{\partial q}{\partial y} \frac{d y}{d t}$

Of the two equations in (9) we find that:

$$
\dot{q}(t)=\frac{\partial p}{\partial x} f_{p}+\frac{\partial q}{\partial y} f_{q}
$$

But: $\frac{\partial q}{\partial x}=\frac{\partial}{\partial x}\left(\frac{\partial z}{\partial y}\right)=\frac{\partial}{\partial y}\left(\frac{\partial z}{\partial x}\right)=\frac{\partial}{\partial y}(p)$

Where for $\quad \dot{q}(t)=\frac{\partial p}{\partial y} f_{p}+\frac{\partial q}{\partial y} f_{q}$

By the end of the equation (2) for $y$ :

$f_{y}+q f_{z}+f_{p} \frac{\partial p}{\partial y}+f_{q} \frac{\partial q}{\partial y}=0$

Or:

$f_{p} \frac{\partial p}{\partial y}+f_{q} \frac{\partial q}{\partial y}=-\left(f_{y}+q f_{z}\right)$

Of the two equations in (14),(15) we find that:

$q^{\prime}(t)=-\left(f_{y}+q f_{z}\right)$

Thus we get the regular differential equivalent system that determines the distribution sector of the equation (2) (Lippold, 1980):

$\grave{x}(t)=f_{p}, \grave{y}(t)=f_{q}, \grave{z}(t)=p f_{p}+q f_{a}$

$\grave{p}(t)=-\left(f_{x}+p f z_{z}\right), \grave{q}(t)=-\left(f_{y}+q f_{z}\right)$

\section{Conclusion}

The conclusion has sought to find out the partial and normative differential equations through the validity of the regular and visual differential equivalent equity and to build the context of the equivalence of the use of numerical and analytical methods, and the search for the use of these equations on the Sports and Physical Side and the importance of those equations, but the focus mainly on the construction of these equations and methods of solve. 
Academic Journal of Research and Scientific Publishing | Vol 3 | Issue 33

Publication Date: 5-1-2022 ISSN: 2706-6495

\section{References:}

Essa, Najiah. (2019). Analytical ways to resolve unrealistically differential equations from the first level, the third annual conference on theories and core and vital sciences.https://www.misuratau.edu.ly/journal/sci/upload/file/R-901-

Conf_3_pages\%20285-298.pdf

Farlow, S. J. (1993). Partial differential equations for scientists and engineers. Courier Corporation.

Hockney, R. W., \& Eastwood, J. W. (2021). Computer simulation using particles. crc Press.

Lippold, G. (1980). Mitchell, AR/Griffiths, DF, The Finite Difference Method in Partial Differential Equations. Chichester-New York-Brisbane-Toronto, John Wiley \& Sons 1980 XII, 272 S.,£ 8.95. ISBN 0-471-27641-3.

Mathews, J. H. (1992). Matlab Programming Guidebook for Numerical Methods.

Morton, K. W., \& Mayers, D. F. (2005). Numerical solution of partial differential equations: an introduction. Cambridge university press.

Othman, A., \& Mahjob, E. (2016). Numerical Solutions of Non-linear Equations With Algorithm Programs (Doctoral dissertation). http://dglib.nilevalley.edu.sd:8080/xmlui/handle/123456789/915.

Press, W. H., Flannery, B. P., Teukolsky, S. A., \& Vetterling, W. T. (1986). Numerical Recipices, the Art of Scientific Computing. Cambridge U. Press, Cambridge, MA.

Smith, G. D., Smith, G. D., \& Smith, G. D. S. (1985). Numerical solution of partial differential equations: finite difference methods. Oxford university press.

Copyright (C) 2022 Turkia Dhawi Alqurashi, AJRSP. This is an Open-Access Article Distributed under the Terms of the Creative Commons Attribution License (CC BY NC)

Doi: https://doi.org/10.52132/Ajrsp.e.2022.33.5 